\title{
LAS COMUNIDADES DE MITILIDOS DEL MEDIOLITORAL ROCOSO DEL DEPARTAMENTO DE LIMA
}

\author{
Carlos Paredes Q. y Juan Tarazona B. \\ Departamento Académico de Ciencias Biológicas \\ Universidad Nacional Mayor de San Marcos
}

\begin{abstract}
SUMARIN
Se estudiaron 34 muestras de los conglomerados de mitilidos de la zona mediolitoral rocosa del Departamento de Lima, (10 $45^{\prime} 38^{\prime \prime} \mathrm{S}$ a $\left.12^{\circ} 57^{\prime} 39^{\prime \prime} \mathrm{S}\right)$, tomadas periodicamente, entre junio y noviembre de 1972 . des:

Utilizando el método del índice biológico, se determinó la existencia de dos comunida-

- Comunidad de Perumytilus purpuratus (Lamarck, 1819), en el horizonte medio de la zona mediolitoral.

- Comunidad de Semimytilus algosus (Gould, 1850), en el horizonte inferior de la zona mediolitoral.

\section{SUMMARY}

Thirty-four samples of mussel ssemblages, were studied in the rocky mid-littoral zone at $10045^{\prime} 38^{\prime \prime}$ S, $12057^{\prime} 39^{\prime \prime}$ S, in the department of Lima, Perí. Such samples were taken periodically, between June and November, 1972. area:

Using the biological index method, two kinds of comunities were determined for the

- Community of Perimytilus purpuratus (Lamarck, 1819), in the mid-horizont of the mid-littoral zone.

- Community of Semimytilus algosus (Gould, 1850), in the lower horizont of the mid-littoral zone.
\end{abstract}

\section{INTRODUCCION}

Hasta la realización de este estudio, las comunidades de mitrlidos que habitan la orilla rocosa de la costa peruana, no habian sido tipificadas cuantitativamente, no existiendo tampoco, uniformidad en cuanto a la terminologfa utilizada para sefialar su distribución vertical en la zona litoral.

Anteriormente (Paredes, 1974) se propuso un modelo de zonación para nuestra orilla rocosa, y sobre esta base, la investigación se orientó hacia la tipificación de las comunidades de mitflidos del mediolitoral rocoso, al conocimiento de su composición cualitativa y cuantitativa así como de su distribución vertical en la zona litoral.

En esta ocasiớn, damos a conocer algunos resultados de los estudios cuantitativos realizados en la zona mediolitoral rocosa del Departamento de Lima, los mismos que forman parte del proyecto "Estudio de las Comunidades Bentónicas del Departamento de Lima".

\section{MATERIAL Y METODOS}

La investigación se llevó a cabo en siete lugares guia distribuídos a lo largo del litoral rocoso del Departamento de Lima, entre los $10^{\circ} 45^{\prime} 38^{\prime \prime} \mathrm{S}$ y los $12^{\circ} 57^{\prime} 39^{\prime \prime} \mathrm{S}$.

Se obtuvieron 17 muestras en el conglomerado de Perumytihs purpuratus (Lamarck, 1819), y otras 17 en el conglomerado de Semimytihus algosus (Gould, 1850), durante el perfodo comprendido entre junio y noviembre de 1972. Además, con fines de comparación, se realizaron observaciones en otros lugares del área de estudio, los que, conjuntamente con los lugares guía, han sido ya señalados en un trabajo anterior (Paredes, 1974).

Las muestras se tomaron periodicamente utilizando un marco do readera de $25 \mathrm{~cm}$ de lado. El material transportado al laboratorio, fue fijado en formol al $6 \%$ o neutralizedo con 
bórax. Posteriormente cada muestra fue tamizada con un tamiz de $1 \mathrm{~mm}$ de abertura, procediéndose luego a separar los ejemplares de las diversas especies. Se obtuvieron los valores de abundancia, frecuencia, presencia y dominancia media, con la finalidad de tipificar las comunidades mediante el cálculo del índice biológico, siguiendo el método de Guille (1970). Para apreciar el valor biocenótico de las especies florísticas, se siguio a Thischler, Bodenheimer y Dajoz (Guille, 1970).

La terminologra utilizada para la zonación, asi como las características fisiográficas y climáticas del área de estudio, fueron discutidas anteriormente (Paredes, 1974).

\section{RESULTADOS}

A. Caracterfsticas de las comunidades de mitilidos de la zona mediolitoral rocosa.

De acuerdó con las observaciones de campo, las comunidades de mitílidos desarrollan en la zona mediolitoral rocosa ocupando los niveles medio e inferior de la orilla rocosa maciza. Solamente en los frentes verticales muy expuestos al oleaje, la comunidad de $P$. purpunatus alcanza el horizonte mediolitoral superior.

Las comunidades se presentan en todas las localidades estudiadas, variando su fisonomia de acuerdo con los factores abioticos predominantes en cada lugar.

El análisis bionómico demuestra que en la zona litoral rocosa existen dos comunidades de mittidos, que se distribuyen verticalmente en la zona mediolitoral, indudablemente, de acuerdo con sus adaptaciones al mayor o menor grado de exposición al aire como consecuencia de la oscilación de las mareas:

1. Comunidad de Perumytilus purpuratus, en el horizonte medio de la zona mediolitoral.

2. Comunidad de Semimytihus algosus, en el horizonte inferior de la zona mediolitoral.

En las orillas de poca pendiente expuestas a la acción de las olas, la comunidad de $P$. purpuratus desarrolla en extensos y gruesos tapices que cubren casi totalmente el sustrato. En estos ambientes las algas son escasas.

Cuando la orilla es de gran pendiente y está muy expuesta al oleaje, la flora casi desaparece, con excepción de Anhfeltia durvillaet, y se forma un grueso estrato de $P$. purpuratus, el que puede alcanzar mas de $10 \mathrm{~cm}$ de espesor.

En orillas protegidas o de oleaje suave, tanto de escasa pendiente como de gran pendiente, la comunidad de $P$. purpuratus se presenta como un delgado tapiz, existiendo, en algunos casos, una variada epiflora que se fija sobre los bivalvos.

La comunidad de $S$. algosus conforma un cinturón o un tapiz mas estrecho y delgado, tanto en las orillas expuestas como en las protegidas. En algunos casos forma manchas aisladas sobre las prominencias rocosas del mediolitoral inferior, habiéndose observado, a veces, alto grado de asociacion con las algas Gymnogongrus furcellatus y Grateloupia doryphora.

\section{B. Antlisis biocenotico.}

1. Comunidad de Perumytilus purpuratus.

a) Flora

Según se presenta en la Tabla 1 , sallaron 16 especies de algas, con predominio de las rojas y verdes.

Resultaron constantes tres especies (Petalonia debilis, Gigartina glomerata y Ceramium rubrufy, 11 fueron comunes y mas 2 especies.

\section{b) Fauna}

Se han hallado 71 especies en esta comunidad (Tabla 2). La Tabla 3 incluye las 29 especies clasificadas según el índice biológico (Ib). 
Las 10 primeras especies de la tabla son preferentes constantes, siendo Perumytilus purpuratus, la que individualiza a la comunidad, por su elevado índice biológico (Ib), frecuencia (F) y dominancia media (Dm).

Las 19 especies restantes son acompañantes, y de acuerdo con los valores de $\mathrm{F}$, se clasifican en acompañantes constantes: Pseudonereis gallapagensis, Typosyllis sp., Dynamella bakeri, Ophiactis Kröyeri. Nereis callaona, Notoplana sp., Halosydna parva, Siphonaria (T.) Lessoni, Littorina peruviana y Tegula (Ch)atra ; y acompaniantes comunes: Lasaea helenae Athyonidium chilensis, Caecum chilense, Callistochiton sp., Fissurella latimarginata (juvenil), Carditella tegulata, Phragmatopoma moerchi, Verruca laevigata e Iselica carotica.

TABLA 1. Frecuencia de las algas en las comunidades de mitnidos de la zona mediolitoral rocosa del Departamento de Lima (17 muestreos en cada comunidad)

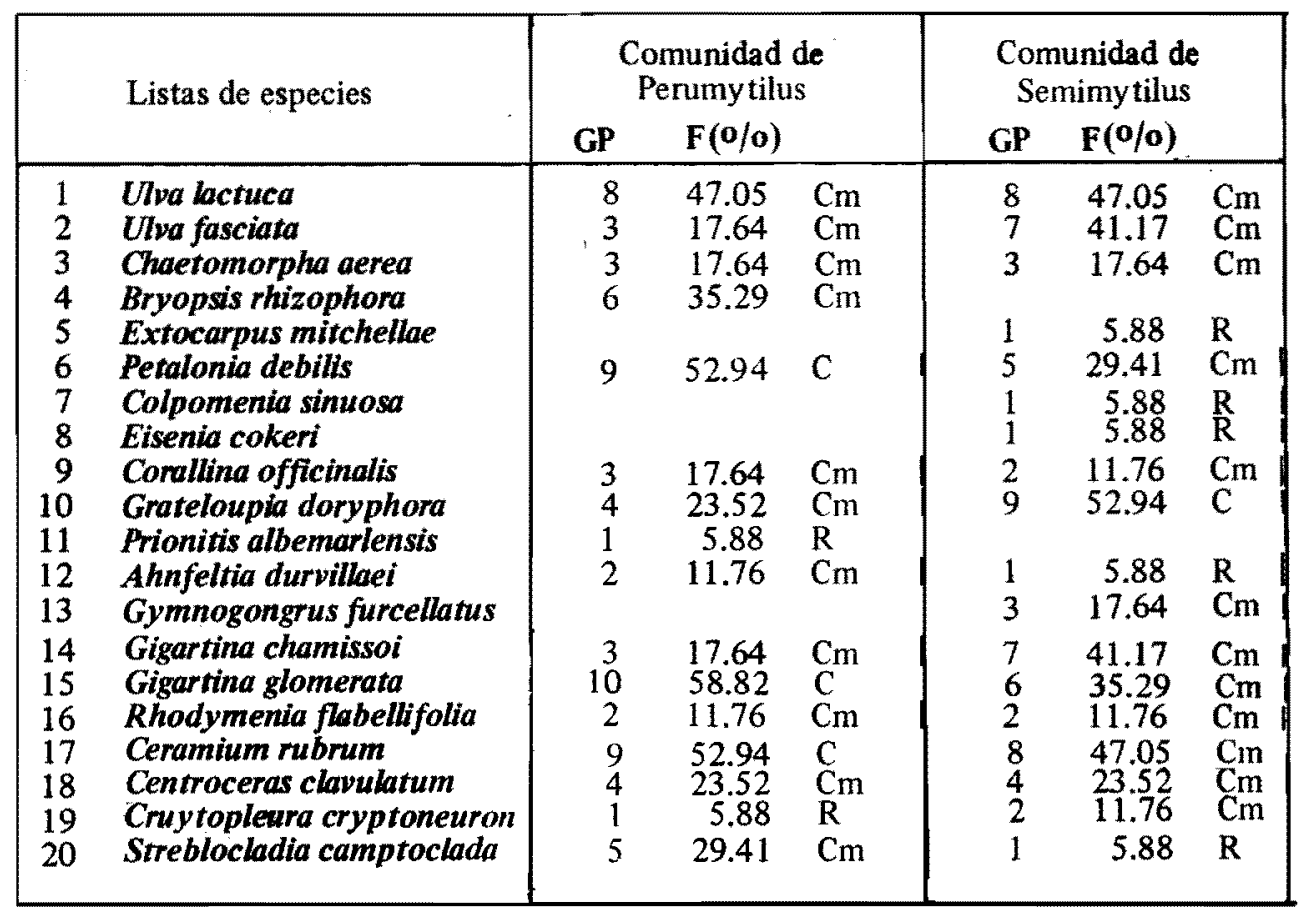

GP; Grado de presencia o frecuencia/total de muestreos; $F(\% / o):$ Frecuencia expreada en porcentaje; C: Constante; Cm: Comun; R: Raro. 
TABLA 2. - Análisis de la comunidad de Perumytilus purpuratus en 17 muestreos

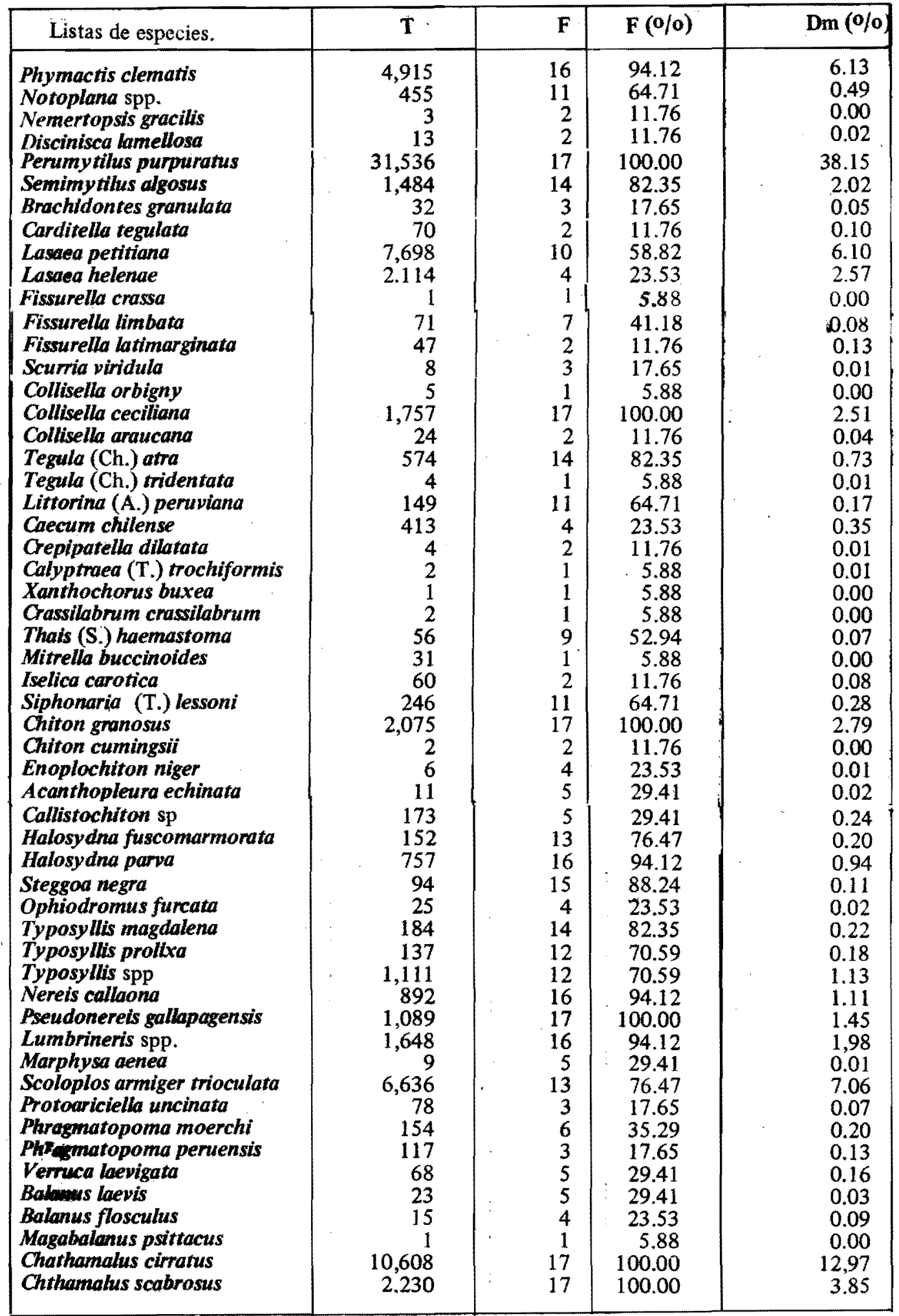




\begin{tabular}{|l} 
Dynamenella bakeri \\
Elasmopus ci. rapax \\
Hyale sp. \\
Allopetrolisthes angulosus \\
Allopetrolisthes spinifrons \\
Petrolisthes granulosus \\
Petrolisthes violaceus \\
Pachycheles crinimanus \\
Acanthocyclus gayi \\
Cyclograpsus cinereus \\
Pachygrapsus transversus \\
Pattalus mollis \\
Athyond dium chilensis \\
Stichaster striatus \\
Tetrapigus niger \\
Ophiactis Kröyeri
\end{tabular}

1,327
271
90
30
6
28
2
26
53
17
11
68
1,866
1
1
452

\begin{tabular}{|r}
16 \\
13 \\
3 \\
2 \\
1 \\
2 \\
1 \\
2 \\
12 \\
1 \\
6 \\
5 \\
3 \\
1 \\
1 \\
11
\end{tabular}

94.12
76.47
17.65
11.76
5.88
11.76
5.88
11.76
70.59
5.88
35.29
29.41
17.65
5.88
5.88
64.71

T: Total de individuos: $F$ : Frecuencia (en 17 muestras): $F(\%)$ : Frecuencia expresada en porcentaje; Dm (o/o): Dominancia media expresada en porcentaje.

De otro lado, el valor biocenótico de las especies clasificadas, puede también considerarse por la distribución porcentual de los grupos taxonómicos, en la que se aprecia un predo. minio de lus Mollusca, Polychaeta y Crustacea (Tabla 4). En cambio, considerando el total de individuos de tedas las especies, se advierte una neta preponderancia numérica de Mollusca (Tabla 4).

\section{Comunidud de Semimytilus algosus.}

a) Flora

Como se aprecia en la Tabla 1, se hallaron 18 aspecies de algas. Resultó constante una sola especie (Grateloupia doryphord, 12 fueron comunes y 5 raras.

b) Fauna

Han sido halladas 59 especies en esta comunidad (Tabla 5). La Tabla 6 incluye las 30 especies clasificadas según el índice biológico (lb).

Las 10 primeras especies clasificadas son preferentes en la comunidad, siendo Semimytilus algosus la que sirve para nominarla, por su mayor indice biológico, frecuencia y dominancia media. De estas especies, 9 son preferentes constantes y sólo Caecum chilense es jreferente común.

Las 20 especies siguientes son acompañantes, y según los valores de $\mathrm{F}$, se clasifican como acompañantes constantes: Protoariciella uncinata, Ophiactis Kröyeri. Typosyllis sp., Elasmopus of rapax, Typosyllis prolixa, Notoplana sp., Dymamenella bakeri, Halosydna parva, H. fuscomarmorata y Collisella ceciliana; y como acompantantes comunes: Athyonidium chilensis, Pattalus mollis, ilyale sp., Phragmatopoma moerchi, Littorina (A) peruviana, Carditelia tegulata, Mitrella buccinoides, Xanthochorus buxea, Ophiodromus furcata y Balanus laevis. 
TABLA 3. Comunidad de Perumytilus purpuratus

\begin{tabular}{|c|c|c|c|c|c|c|c|c|c|c|c|c|c|c|c|c|}
\hline & \multirow[t]{2}{*}{ Especies } & \multicolumn{8}{|c|}{ Clasificación } & \multirow[b]{2}{*}{9} & \multirow[b]{2}{*}{10} & \multirow[b]{2}{*}{$\mathbf{F}$} & \multirow[b]{2}{*}{$\mathbf{P}$} & \multirow[b]{2}{*}{$\mathbf{l b}$} & \multirow[b]{2}{*}{ Dm } & \multirow[b]{2}{*}{ Dc } \\
\hline & & 1 & 2 & 3 & 4 & 5 & 6 & 7 & 8 & & & & & & & \\
\hline 1 & Perumytilus purpuratus & 13 & 2 & 1 & 1 & & & & & & & 17 & 17 & 163 & 38.15 & 38.15 \\
\hline 2 & Chthamahus cirratus & & 7 & 4 & 4 & 1 & & 1 & & & & 17 & 17 & 132 & 12.97 & 51.12 \\
\hline 3 & Chiton granosus & & 1 & 1 & 1 & 3 & 4 & & 1 & 2 & & 17 & 13 & 69 & 2.79 & 53.91 \\
\hline 4 & Scoloplos armiger & 2 & 1 & 2 & 2 & 1 & & & 1 & & & 13 & 9 & 68 & 7.06 & 60.97 \\
\hline 5 & Phymactis clematis & & 2 & 3 & 1 & 1 & & 2 & & 2 & & 16 & 11 & 67 & 6.13 & 67.10 \\
\hline 6 & Chthamalus scabrosus & & 1 & 2 & & 1 & 3 & 1 & & & 1 & 17 & 9 & 51 & 3.85 & 70.95 \\
\hline 7 & Collisella ceciliana & & & & 1 & 1 & 3 & 2 & 4 & & 2 & 17 & 13 & 50 & 2.51 & 73.46 \\
\hline 8 & Lasaea petitiana & 1 & 1 & 2 & & 1 & & & & & 1 & 10 & 6 & 42 & 6.10 & 79.56 \\
\hline 9 & Lumbrineris spp. & & & & & 2 & 3 & & 4 & 1 & & 16 & 10 & 41 & 1.98 & 81.54 \\
\hline 10 & Semimy tilus algosus & & 1 & 1 & 1 & 1 & & & & 3 & & 14 & 7 & 36 & 2.02 & 83.56 \\
\hline 11 & Lasaea helenae & & 2 & 1 & 1 & & & & & & & 4 & 4 & 33 & 2.57 & 86.13 \\
\hline 12 & Pseudonereis gallapagensis & & & & & & 1 & 2 & 1 & 3 & 4 & 17 & 11 & 26 & 1.45 & 87.58 \\
\hline 13 & Typosyllis sp. & & & & 1 & & 1 & 2 & 1 & 1 & 1 & 12 & 7 & 26 & 1.13 & 88.71 \\
\hline 14 & Dynamenella bakeri & & & & & 1 & 1 & 2 & & 2 & 2 & 16 & 8 & 25 & 1.73 & 90.44 \\
\hline 15 & Athyonidium chilensis & 1 & & & & 1 & & & & & & 3 & 2 & 16 & 1.98 & 92.42 \\
\hline 16 & Ophiactis Kröyeri & & & & 1 & 1 & & & 1 & & & 11 & 3 & 16 & 0.84 & $93: 26$ \\
\hline 17 & Nereis callaona & & & & 1 & 1 & & & & & 2 & 16 & 4 & 15 & 1.11 & 94.37 \\
\hline 18 & Notoplana sp. & & & & & & & 1 & 1 & 1 & & .11 & 3 & 9 & 0.49 & 94.86 \\
\hline 19 & Caecum chilense & & & & 2 & & & & & & & 4 & 2 & 7 & 0.35 & 95.21 \\
\hline 20 & Halosydna parva & & & & & & & 1 & & & 2 & 16 & 3 & 6 & 0.94 & 96.15 \\
\hline 21 & Siphonaria (T.) lessoni & & & & & 2 & & & & & & 11 & 2 & 6 & 0.28 & 96.43 \\
\hline 22 & Littorina (A.) peruviana & & & & & & & & 2 & & & 11 & 2 & 6 & 0.17 & 96.60 \\
\hline 23 & Callistochiton sp. & & & & & & & & 1 & 1 & & 5 & 2 & 5 & 0.24 & 96.84 \\
\hline 24 & Tegula (Ch.) atra & & & & & & & 1 & & & & 14 & 1 & 4 & 0.73 & 97.57 \\
\hline 25 & Fissurella latimarginata & & & & & & & $i$ & & & & 2 & 1 & 4 & 0.13 & 97.70 \\
\hline 26 & Carditella tegulata & & & & & & & 1 & & & & 2 & 1 & 4 & 0.10 & 97.80 \\
\hline 27 & Phragmatopoma moerchi & & & & & & & & & & 1 & 6 & 2 & 1 & 0.20 & 98.00 \\
\hline 28 & Verruca laevigata & & & & & & & & & & 1 & 5 & 2 & 1 & 0.16 & 98.16 \\
\hline 29 & Iselica carotica & & & & & & & & & & 1 & 2 & 1 & $i$ & 0.08 & 98.24 \\
\hline
\end{tabular}

F; Frecuencia; P: Presencia; Ib: Indice biológico; Dm: Dominancia media; Dc: Dominancia acumulada. 
TABLA 4. Porcentaje de especies y de individuos de los diversos grupos zoológicos, en relación al número total de especies y de individuos presentes en cada comunidad.

\begin{tabular}{|l|c|c|c|c|}
\hline \multirow{2}{*}{ Grupos } & \multicolumn{4}{|c|}{ c o m u n i d a d e s } \\
\cline { 2 - 5 } & \multicolumn{2}{|c|}{$P$. purpurarus } & \multicolumn{2}{c|}{ S. algosus } \\
\cline { 2 - 5 } & Espec. & Ind. & Espec. & Ind. \\
\hline \multirow{2}{*}{ Mollusca } & 42.25 & 57.94 & 42.37 & 66.00 \\
Polychaeta & 21.13 & 15.52 & 25.42 & 21.40 \\
Crustacea & 23.94 & 17.56 & 22.03 & 2.78 \\
Echinodermata & 7.04 & 2.84 & 6.78 & 5.30 \\
Anthozoa & 1.41 & 5.83 & 1.69 & 2.52 \\
Turbellaria & 1.41 & 0.54 & 1.69 & 0.56 \\
Nemertea & 1.41 & 0.00 & 0.00 & 0.00 \\
Brachiopoda & 1.41 & 0.01 & 0.00 & 0.00 \\
\hline
\end{tabular}


TABLA 5.- Análisis de la comunidad de Semimytilus algosus en 17 muestreos.

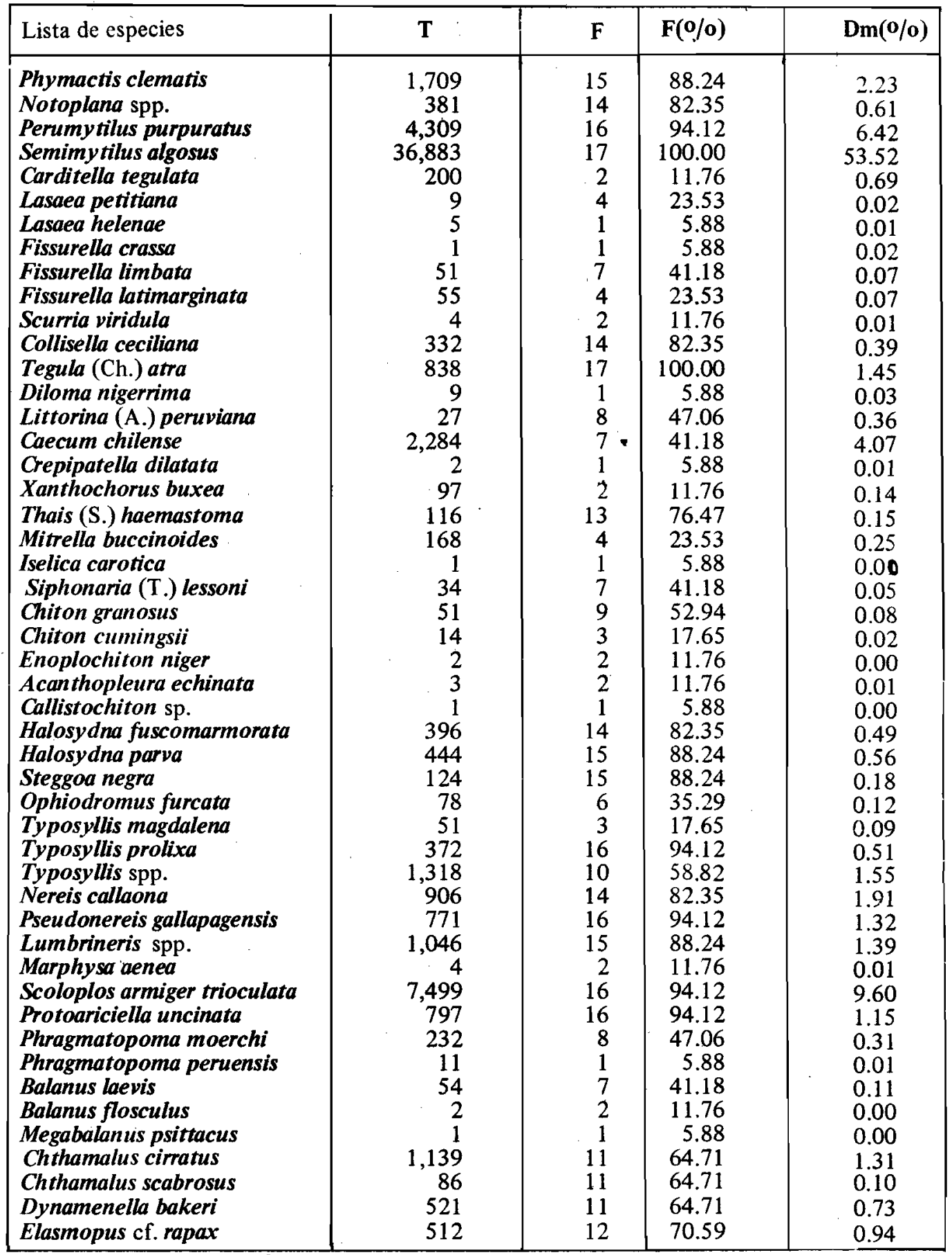




\begin{tabular}{|l|r|r|r|r|} 
Hyale sp. & 218 & 6 & 35.29 & 0.39 \\
Puchycheles crinbanus & 1 & 1 & 5.88 & 0.00 \\
Acanthocyclus gayi & 21 & 8 & 47.06 & 0.00 \\
Pachygrapsus transversus & 3 & 3 & 17.65 & 0.00 \\
Pilumnoides perlatus & 7 & 4 & 23.53 & 0.01 \\
Acanthonyx petiveri & 2 & 2 & 11.76 & 0.01 \\
Pattalus mollis & 1,846 & 5 & 29.41 & 3.86 \\
Athyonidium chilensis & 308 & 3 & 17.65 & 0.66 \\
Stichaster striatus & 6 & 4 & 23.53 & 0.01 \\
Ophiactis kröyeri & 1,432 & 14 & 82.35 & 1.60 \\
\hline
\end{tabular}

T: Total de individuos; F: Frecuencia (en 17 muestras); $\mathrm{F}(\%)$ ): Frecuencia expresada en porcentaje; $\mathrm{Dm}(\%)$ : Dominancia media expresada en porcentaje.

En esta comunidad se observa, al igual que en la de $P$. purpuratus, una distribución porcentual de la fauna con predominio de los Mollusca, Polychacta y Crustacea (Tabla 4). En cambio, numéricamente, la población de la comunidad se distribuye con una mayor dominancia de los Mollusca, en comparación con la comunidad de $\boldsymbol{P}$. purpuratus.

3. Comparación entre las dos comunidades.

Del estudio de las tablas 3 y 6 , se advierte que entre las especies preferentes constantes, 6 de ellas son las mismas en las dos comunidades, siendo el valor del $\mathrm{Ib}$, el que nos permite señalar el grado de preferencia; asi, Chthamalus cirratus es altamente preferente en la comunidad de $\boldsymbol{P}$. purpuratus $\boldsymbol{y}$ Scoloplos armiger trioculatalo es en la comunidad de $\boldsymbol{S}$. algosus. En cambio, Phymactis clematis (juvenil) y Lumbrineris sp., son especies que tienen casi un mismo valor biocenótico como integrantes de las comunidades.

De otro lado, hay marcadas diferencias entre ambas comunidades; así, Chiton granosus, Chthamalus scabrosus y Lasaea petitiana, especies preferentęs de la comunidad de $P$. purpuratus, sólo resultaron accesorias en la comunidad de $S$. algosus, es decir que no tienen índice biológico.

La especie Collisella ceciliana, preferente en la comunidad de $\boldsymbol{P}$. purpuratus, sólo es acompañante en la comunidad de $S$. algosus.

Tegula (Ch.) atra, Nereis callaona, Caecum chilense y Pseudonereis gallapagensis, especies preferentes en la comunidad de $S$. algosus, son acompañantes en la comunidad de $P$. purpuratus.

Por otra parte, en la comunidad de $P$. prupuratus se han registrado 15 especies que están ausentes en la comunidad de $S$. algosus, las mismas que, por carecer de índice biológico, no tienen mayor significado en la composición faunistica de ambas comunidades, debiendo considerarse enteramente accesorias. Estas especies son: Nemertopsis gracilis, Discinisca lamellosa, Brachidontes granulata, Collisella araucana, Verruca laevigata, Allopetrolisthes angulosus, Petrolisthes granulosus, Collisella orbignyi, Tegula (Ch.) tridentada, Calyptraea (T.) trochiformis, Crassilabrum crassilabrum, Allopetrolisthes spinifrons, Petrolisthes violaceus, Cyclograpsus cinereus y Tetrapigus niger.

Finalmente, considerando el ordenamiento de las especies clasificadas como acompañantes en ambas comunidades se observan marcadas diferencias, como puede apreciarse en las Tablas 3 y 6 . 
TABLA 6. Comunidad de Semimytilus algosus

\begin{tabular}{|c|c|c|c|c|c|c|c|c|c|c|c|c|c|c|c|}
\hline \multirow[b]{2}{*}{ Especies } & \multicolumn{10}{|c|}{ Clasificación } & \multirow[b]{2}{*}{$\mathbf{F}$} & \multirow[b]{2}{*}{$\mathbf{P}$} & \multirow[b]{2}{*}{ Ib } & \multirow[b]{2}{*}{ Dm } & \multirow[b]{2}{*}{ Dc } \\
\hline & 1 & 2 & 3 & 4 & 5 & 6 & 7 & 8 & 9 & 10 & & & & & \\
\hline 1 Semimytilus algosus & 15 & 2 & & & & & & & & & 17 & 17 & 168 & 53.52 & 53.52 \\
\hline 2 Scoloplos armiger & & 6 & 6 & & 2 & & & 1 & & & 16 & 15 & 117 & 9.60 & 63.12 \\
\hline 3 Perumytilus purpuratus & & 5 & 1 & 1 & 1 & 2 & & & 1 & 1 & 16 & 12 & 79 & 6.42 & 69.54 \\
\hline 4 Tegula (Ch.) atra & & & & & 4 & 2 & 3 & & 1 & & 17 & 10 & 48 & 1.45 & 70.99 \\
\hline 5 Nereis callaona & & 1 & 1 & & & & 2 & 2 & 1 & & 14 & 9 & 47 & 1.91 & 72.90 \\
\hline 6 Lumbrineris spp. & & & & & 2 & 2 & 2 & 4 & & 1 & 15 & 11 & 43 & 1.39 & 74.29 \\
\hline 7 Coecum chilense & 1 & 1 & 1 & 1 & & & & & & 1 & 7 & 5 & 35 & 4.07 & 78.36 \\
\hline 8 Pseudonereis gallapagensis & & & 1 & 1 & 1 & 1 & 1 & 1 & & 1 & 16 & 7 & 34 & 1.32 & 79.68 \\
\hline 9 Phymactis clematis & & 1 & 1 & 1 & & 1 & & 1. & & 1 & 15 & 6 & 33 & 2.23 & 81.91 \\
\hline 10 Chthamalus cirratus & & & & 2 & 2 & 1 & & & & 1 & 11 & 6 & 32 & 1.31 & 83.22 \\
\hline 11 Protoariciella uncinata & & & & & 1 & 1 & 2 & 1 & 4 & 2 & 16 & 11 & 32 & 1.15 & 84.37 \\
\hline 12 Ophiactis Kröyeri & & & 1 & 2 & & 1 & & & 1 & 1 & 14 & 6 & 30 & 1.60 & 85.97 \\
\hline 13 Typosyllis spp. & & & 2 & & 1 & & & 2 & & & 10 & 5 & 28 & 1.55 & 87.52 \\
\hline 14 Athyonidium chilensis & & 1 & 1 & & & 1 & & & & & 3 & 3 & 22 & 0.66 & 88.18 \\
\hline 15 Pattalus mollis & 1 & & & 1 & & & & 1 & & & 5 & 3 & 20 & 3.86 & 92.04 \\
\hline 16 Elasmopus cf. rapax & & & 1 & & & 1 & & 1 & 1 & 2 & 12 & 6 & 20 & 0.94 & 92.98 \\
\hline 17 Typosyllis prolixa & & & & & 1 & 1 & 1 & 1 & 1 & & 10 & 5 & 20 & 0.51 & 93.49 \\
\hline 18 Notoplana sp. & & & & 1 & 1 & & 1 & & & & 14 & 3 & 17 & 0.61 & 94.10 \\
\hline 19 Dynamenella bakeri & & & 1 & 1 & & & & & & 1 & 11 & 3 & 16 & 0.73 & 94.83 \\
\hline 20 Halosydna parva & & & & & 1 & & 2 & & & 2 & 15 & 5 & 16 & 0.56 & 95.39 \\
\hline 21 Hyale sp. & & & & 1 & & & 1 & & 1 & & 6 & 3 & 13 & 0.39 & 95.78 \\
\hline 22 Phragmatopoma moerchi & & & & 1 & & . & & & 2 & 2 & 8 & 5 & 13 & 0.31 & 96.09 \\
\hline 23 Littorina (A.) peruviana & & & & 1 & & 1 & & & & & 8 & 2 & 12 & 0.36 & 96.45 \\
\hline 24 Halosydna fuscomarmorata & & & & & & & & 2 & 2 & & 14 & 4 & 10 & 0.49 & 96.94 \\
\hline 25 Collisella cecillana & & & & & & 1 & 1 & & & 1 & 14 & 3 & 10 & 0.39 & 97.33 \\
\hline 26 Carditella tegulata & & & & 1 & & & & & & & 2 & 1 & 7 & 0.69 & 98.02 \\
\hline 27 Mitrella buccinoides & & & & & & 1 & & & & & 4 & 1 & 5 & 0.25 & 98.27 \\
\hline 28 Xanthochorus buxea & & & & & & & 1 & & & & 2 & 1 & 4 & 0.14 & 98.41 \\
\hline 29 Ophiodromus furcata & & & & & & & & & 1 & & 6 & 1 & 2 & 0.12 & 98.53 \\
\hline 30 Balanus laevis & & & & & & & & & 1 & & 7 & 1 & 2 & 0.11 & 98.64 \\
\hline
\end{tabular}

F; Frecuencia; P: Presencia; Ib: Indice biológico; Dm: Dominancia media; Dc: Dominancia 


\section{DISCUSION}

Hasta la realización del presente estudio, las comunidades de mitilidos que habitan la orilla rocosa de la costa peruana, no habian sido definidas cuantitativamente. Así, Vegas (1963) refiere a los mitilidos como integrantes de la Zona de Littorina; Huamán (1967) los menciona dentro del Piso de Balanidos; y Livia (1971) los considera integrantes de la biocenosis del medio litoral rocoso.

En Argentina, P. purpuratus (referido como Brachidontes purpuratus) ha sido citado, caracterizando la comunidad del "mejillín" o "mejillinar" del medio litoral (Ringuelet y col., 1962; Kuhnemann, 1969, 1971). Olivier y col, (1966a), empleando métodos biocenóticos, hallan en el mesolitoral rocoso de Mar del Plata, una biocenosis de Mytilus platensis- Brachidontes rodriguezi; en cambio, en Puerto Pardelas (1966b), diferencian en el horizonte mediolitoral medio, una comunidad de $B$. purpuratus; y en el horizonte mediolitoral inferior, una comunidad de Mytilus chilensis- Aulacomya magellanica.

En . Chile, Lopez y Osorio (1977) analizaron la diversidad específica y la abundancia en cinco muestras de la zona fitoral de Putemun, hallando que $\boldsymbol{P}$. purpuratus domina en el nivel superior, y Mytilus chilensis en el inferior.

En el presente trabajo, empleando el método del índice biológico, se ha encontrado que en el mediolitoral rocoso del Departamento de Lima, los conglomerados de mitilidos están conformados por una comunidad de $\boldsymbol{P}$. purpuratus en el mediolitoral medio, y otra de $\boldsymbol{S}$. al . gosus, en el mediolitoral inferior. Sin duda, el factor abiótico preponderante para esta división, es el grado de inmersión y la consiguiente exposición al aire por causa de la oscilación de las mareas.

En cuanto al rango de distribución vertical de las especies caracteristicas, es de notar que $\boldsymbol{P}$. purpuratus alcanza el nivel de la comunidad de $S$. algosus, ocupando el tercer lugar entre las especies preferentes, pero con una densidad escasa (Tablas 3 y 5 ). El mismo fenómeno ha sido observado en Chile, (Romo y Alveal, 1977) y en Argentina (Zaixso y Pastor, 1977).

Igualmente, $\boldsymbol{S}$. algosus alcanza el nivel de la comunidad de $\boldsymbol{P}$. purpuratus, ocupando el décimo lugar entre las especies preferentes, pero con una densidad disminuida, situación que indica su poca adaptación para soportar un mayor porcentaje de emersión (Tablas 4 y 6 ).

Livia (1971) realizó un estudio en algunos lugares del Departamento de Lima, y en base a los resultados del análisis de 5 muestras del horizonte superior del piso mediolitoral (equivalente a la comunidad de $\boldsymbol{P}$. purpuratus), y 5 del horizonte inferior (equivalente a la comunidad de $S$. algosus), señalo como especies características del horizonte superior a $P$. purpuratus y Chthamalus cirratus, y como accidental a $S$. algosus; en cambio, en el presente trabajo, Ch. cirratus y $\mathbf{S}$. algosus resultan clasificadas entre las 10 especies preferentes de la comunidad.

En el horizonte inferior, Livia (1971), señala como especie característica a $S$. algosus y como accidental a $P$. purpuratus, especie que, en nuestro caso, ocupa uno de los primeros lugares como preferente.

Teniendo en cuenta la composición y las especies características, las comunidades del mediolitoral rocoso estudiadas por Ringuelet y col. (1962) y Olivier y col, (1966a

y b), pueden considerarse equivalente a las comunidades de mitrididos de nuestra zona mediolitoral.

Mayor similitud se representa con los hallazgos de Lopez y Osorio (1977) en la costa chilena (420 $24^{\prime} 40^{\prime \prime} \mathrm{S}, 730^{\circ} 44^{\prime} 40^{\prime} \mathrm{W}$ ), en donde Mytilus chilensis ocupa el nivel co. rrespondiente a la comunidad de $S$. algosus.

En latitudes más bajas ( $32044^{\prime} \mathrm{S}, 71031^{\prime} \mathrm{W}$ ), comprendidas dentro de la Provincia Peruano-Chilena, las comunidades $\boldsymbol{P}$. purpuratus y $\boldsymbol{S}$. algosus, estudiadas por Romo y Alveal (1977), presentan, prácticamente, la misma composición y distribución vertical que las estudiadas en este trabajo.

Finalmente, en to que respecta a la distribución porcentual de los grupos taxonómicos que integran las comunidades de mitílidos de la zona mediolitoral del Departamento de Lima. se encontró un predominio de Mollusca, Polychaeta y Crustacea, lo cual coincide con lo reportado en áreas vecinas Zaixso y Pastor, (1977); Lopez y Osorio, (1977), Romo y Al-
veal, (1977). 


\section{CONCLUSIONES}

1. El estudio de los conglomerados de mitilidos que habitan la zona mediolitoral rocosa del Departamento de Lima, empleando el método biocenótico del índice biológico, permite concluír, que se encuentran conformados por dos comunidades:

- Comunidad de Perumytilus purpurarus.

- Comunidad de Semimytilis algosus.

2. La comunidad de Perumytilus purpuratus se localiza en el horizonte mediolitoral medio, en orillas protegidas, $y$ puede extenderse al horizonte mediolitoral superior, en orillas muy expuestas. Entre los organismos preferentes de esta comunidad, destacan en la fauna, Chthamalus cirratus, Chiton granosus (juvenal), Chthamalus scabrosus, Collisella ceciliana y Lasaea petitiana; y entre las algas, son constantes Petalonia debilis, Gigartina glomerata y Ceramium rubrum.

3. La comunidad de Semimytilus algosts ocupa el horizonte mediolitoral inferior, teniendo como especies faunísticas de mayor indice biológico a Scoloplos armiger trioculata, $P$. purpuratus, Tegula (Ch.) atra (juvenil) y Nereis calloona; y, como alga constante a Gra. teloupia doryphora.

\section{AGRADECIMIENTOS}

Agradecemos al Dr. César Acleto O. por la determinación específica de las algas, y al personal del Laboratorio de Zoología General del Departamento Académico de Ciencias Biológicas, por su valiosa ayuda, tanto en los trabajos de campo como en el análisis de las muestras.

\section{REFERENCIAS BIBLIOGRAFICAS}

ALVEAL, K. 1970. Estudios ficoecológicos en la región costera de Valparaíso. Rev. Biol. mar, $14(1): 1 \cdot 88$

GUILLE, A. 1970. Bionomie benthique du plateu continental de la cóte catalana francaise. II. Lès communautés de la macrofaune. Vie et Milieu. 21 (1B) : $149 \cdot 280$.

HUAMAN, H. 1967. Observaciones bioecológicas de algunas playas rocosas del sur de Lima. Tesis de Bachiller. Univ. Nac. Mayor de San Marcos. Lima, Perú.

KUHNEMANN, O. 1969. Observaciones acerca de los límites del Piso Mesolitoral en el Dominio Atlấntico Austral Americano. Physis, 28 (77) : 331 - 349

1971. Vegetación marina de la Ría de Puerto Deseado. CIBIMA, Contr. Cient. No $30,125 \mathrm{pp}$.

LIVIA, A. 1971. Contribución al estudio de la Biocenosis del mediolitoral rocoso de playas cercanas a Lima. Tesis de Bachiller. Univ. Nac. Mayor de San Marcos. Lima, Perú.

LOPEZ, M. y C. OSORIO. 1977. Diversidad biológica en la comunidad intermareal de Putemun. Chiloe. Bol. Soc. Biol. de Concepción. 51 (1) : 123 - 127.

OLIVIER; S. R., I. KREIBHOM y R. BASTIDA. 1966a. Estudios biocenóticos en las costas de Chubut (Argentina). I. Zonación biocenológica de Puerto Pardelas (Golfo Nuevo). Bol. Inst. Biol. Mar. 10:1 - 74

OLIVIER, S. R., A. ESCOFET, J. ORENSANZ, S. PEZZANI, A. TURRO y M. TURRO. 1966b. Contribución al conocimiento de las comunidades bénticas de Mar del Plata. An. Com. Invest. Cient. $7: 185-206$.

PAREDES, C. 1974. El modelo de zonación en la orilla rocosa del Departamento de Lima. Rev. Per. Biol. 1 (2) : 166 - 191. 
RINGUELET, R., A. AMOR, N. MAGALDI y R. PALLARES. 1962. Estudio ecológico de la fauna intercotidal de Puerto Deseado en Febrero de 1961 (Santa Cruz, Argentina). Physis, 23 (64) : 35 - 53.

ROMO, H. y K. ALVEAL. 1977. Las comunidades del litoral rocoso de Punta Ventanilla Bahía de Quintero-Chile. Gayana Misc. No 6, 40 pp.

VEGAS, M. 1963. Contribución al conocimiento de la Zona de Littorina de la Costa Peruana. An. Cient. U. A. 1 (2) : 174 - 193.

ZAIXSO, H. y C. PASTOR. 1977. Observaciones sobre la ecología de los mitilidos de la Ría Deseado. I. Distribución y análisis biocenótico. CIBIMA, Contr. Cient. No $131: 46$ pp. 